\title{
The origin and evolution of dust belts
}

\author{
Mark C. Wyatt \\ UK Astronomy Technology Centre, Royal Observatory, Edinburgh EH9 3HJ, UK \\ email: wyatt@roe.ac.uk
}

\begin{abstract}
Planetary systems are made up of objects with sizes ranging from gas giant planets down to asteroids and on to micron sized dust. Dust in the zodiacal cloud in the solar system originates in the break-up of asteroids and comets and then migrates in toward the Sun due to P$\mathrm{R}$ drag. The dynamical evolution of the dust is also affected by the gravitational perturbations of the solar system's planets, and the consequence of those perturbations is evident in the asymmetric and clumpy structure of the zodiacal cloud. In the last couple of years features in the cloud have been identified with asteroid collisions which occurred just a few Myr ago implying that steady state models for the zodiacal cloud will have to be reconsidered. Many extrasolar systems also harbor massive dust belts and the structures of those dust belts have been linked to perturbations from unseen planets. This paper reviews the dominant physical processes affecting the evolution of dust grains and describes the techniques which have been developed to model their dynamics and identify the sources of dust structures in both the solar system and extrasolar systems.
\end{abstract}

Keywords. Celestial mechanics; methods: numerical; interplanetary medium; circumstellar matter

\section{Dust in the Solar System}

The inner solar system is populated with a large number of dust grains which lie in a disk around the Sun in which the Earth is embedded. This disk is known as the Zodiacal Cloud (ZC) and it can be seen with the naked eye: diffuse emission, known as the zodiacal light, is seen near the Sun just before sunrise or after sunset and comes from sunlight scattered by the dust grains. The ZC is even more readily detected in the infrared where it is the brightest object in the sky once outside the Earth's atmosphere (Low et al. 1984; Kelsall et al. 1998). Such wavelengths probe thermal emission from the dust grains which are heated by the Sun.

The dust has a very short lifetime and so must be constantly replenished. There are several possible sources for this replenishment. The dust could come from the break-up of large bodies in the solar system, such as from collisions between asteroids in the Asteroid Belt $(\mathrm{AB})$, the sublimation or collisional disintegration of comets, or from the collisional destruction of more distant bodies such as those in the Kuiper Belt (KB). The dust could also come from outside the solar system, i.e., from the interstellar medium (ISM). All of these sources contribute to the $\mathrm{ZC}$ to some extent, but the contribution of the last two is thought to be low, because dust originating in the KB is scattered by Jupiter before it reaches the inner solar system (Moro-Martín \& Malhotra 2002) and the Sun is currently in a tenuous region of the ISM called the local bubble (Grogan et al. 1996). The relative contribution of asteroids and comets to the $\mathrm{ZC}$ is still debated (e.g., Sykes et al. 1986; Kortenkamp \& Dermott 1998), but at least 30\% of the ZC dust has been linked to asteroids (Grogan et al. 2001), so the following sections explain the theory behind the structure of the $\mathrm{ZC}$ and how it is modeled using the $\mathrm{AB}$ as the main example. For a more detailed discussion the reader is referred to Dermott et al. (2001). 


\subsection{Large Scale Structure}

The AB is a collisional cascade in which large asteroids are continually colliding and getting broken up into smaller fragments. This is the reason the size distribution of the detected members (Durda \& Dermott 1997) is close to the theoretical value expected in such a cascade, $n(D) \propto D^{2-3 q}$ where $q=11 / 6$ (Dohnanyi 1969). While asteroids smaller than a few $\mathrm{km}$ have not been detected in the $\mathrm{AB}$, the cascade is believed to extend down to $\mu \mathrm{m}$-sized dust particles. It is those smallest particles that are detected in the infrared, because such observations are sensitive to the surface area of the cascade, not its mass which is in the largest asteroids. It is difficult to determine how much dust is created in this cascade, because the extrapolation of this size distribution down to small sizes depends on details of collisional processes we cannot be sure of (Durda et al. 1998).

For most of the cascade, gravity is the dominant force acting on its members. Small dust, however, is affected by its interaction with solar radiation (Burns et al. 1979; Gustafson 1994). The resulting force falls off $\propto r^{-2}$, so it is commonly defined by the ratio $\beta$, of its magnitude to that of gravity. Different size dust grains are affected to a different extent by radiation forces, and for large grains the approximation $\beta \propto 1 / D$ is valid. There are two components to the force: a radial component, known as radiation pressure, which means that dust grains effectively see a smaller Sun so that their orbits are more elliptical than that of the parent asteroids and grains with $\beta>0.5$ (corresponding to those $<1 \mu \mathrm{m}$ in the solar system) are blown out on hyperbolic orbits as soon as they are created; and a tangential component, known as Poynting-Robertson (P-R) drag, which makes dust particle orbits spiral into the Sun, where the particles evaporate, at a rate $\dot{r}_{\mathrm{pr}}=-2 \alpha / r$ for initially eccentric orbits, where $\alpha=6.24 \times 10^{-4} \beta M_{\star} \mathrm{AU}^{2} \mathrm{yr}^{-1}$.

The large scale structure of the ZC is determined by the balance of collisions and P-R drag. The continuity equation expressing this balance can be solved analytically if you assume the dust created in the $\mathrm{AB}$ is of the same size, since the collisional lifetime of this dust is then just $t_{\text {per }} / 4 \pi \tau_{\text {eff }}$, where $\tau_{\text {eff }}$ is the effective optical depth of these grains and $t_{\text {per }}$ is their orbital period (Wyatt 1999). The resulting dust distribution depends only on the factor $\eta_{0}=5000 \tau$ eff $\left(r_{0}\right) \sqrt{r_{0} / M_{\star}} / \beta$, which is determined by the rate at which the dust produced. In this model, if the $\mathrm{AB}$ was very dense, and the production rate was high $\left(\eta_{0} \gg 1\right)$, the dust would be destroyed in mutual collisions before making it into the inner regions of the solar system. However, the AB is relatively tenuous $\left(\eta_{0} \ll 1\right)$ implying that few collisions should occur and the dust distribution should have a surface density which is constant with distance from the Sun.

In reality dust is produced with a range of sizes, and it is clear that while the largest grains remain in the $\mathrm{AB}$ their whole lifetime and small grains make it to the Sun without suffering a collision, some grains only complete part of the journey and are then destroyed. While the continuity equation can be solved numerically to derive the resulting size distribution, simple arguments show that the size distribution expected in such a situation should peak at the size for which the collisional lifetime is equal to the P-R drag lifetime (Wyatt et al. 1999; Dermott et al. 2001). Since this is a few $100 \mu \mathrm{m}$ for dust originating in the $\mathrm{AB}$ (particles for which collisional and P-R drag lifetimes are $\sim 4$ Myr, Wyatt et al. 1999; also Leinert \& Grün 1990), this is in good agreement with the peak in the size distribution of dust accreted by the Earth found by LDEF (Love \& Brownlee 1993).

\subsection{Dust Bands}

The small scale structure of the ZC is particularly telling about the sources of its dust grains. One such feature is the dust bands which were discovered by IRAS in 1984 (Low et al. 1984). IRAS produced scans through the ZC perpendicular to the ecliptic. As 
expected the brightness increases to a peak in the ecliptic, falling off on either side. However, there are also shoulders of emission at $\pm 10^{\circ}$ and $\pm 2^{\circ}$ ecliptic latitude. One method of isolating the dust band features is to use Fourier filtering to remove the low frequency background component. When applied to the database of IRAS scans, such analysis shows that the structure of the dust bands varies significantly throughout the year as the Earth moves around its orbit; it is also different when viewed in front of and behind the Earth as well as in different wavebands (Fig. 1a; Grogan et al. 1997).

The latitudes of the bands meant they were quickly identified with the Hirayama asteroid families (Dermott et al. 1984), because the distribution of proper inclinations $\left(I_{p}\right)$ in the $\mathrm{AB}$ has two peaks, one at $\sim 10^{\circ}$ from the Eos family and one at $\sim 2^{\circ}$ from the Themis and Koronis families. The reason these families would be expected to produce dust bands is that asteroids on inclined orbits spend a disproportionate amount of time at large heights above the ecliptic plane. P-R drag has no effect on the orbital planes of dust produced by these families, so their distribution would be expected also to peak at large heights above the ecliptic at all distances from the Sun. The observing geometry means that when looking through this dust we would expect to see peaks at latitudes of $\pm I_{p}$.

However, that idealized model for the dust bands cannot properly account for their seasonal variation. This is because while the orbital planes of the dust grains are not affected by P-R drag, these are significantly affected by the secular perturbations of the planets. The secular perturbation equations can be solved in the presence of P-R drag to show that their effect is to make both the inclinations $y=I \exp i \Omega$ and eccentricities $z=e \exp i \tilde{\omega}$ of dust grains precess around circles centered on forced elements. However, the centers of these circles are found to be different for different particle sizes and must be determined by integrating over the particle's evolutionary history. This led Dermott et al. (1992) to invent the particle in a circle method of determining the orbital element distributions of different sized particles originating from different families. This method followed the evolution of waves of different sized particles started at some point in the past on circles in the $\mathrm{z}$ and $\mathrm{y}$ planes defined by the orbital elements of the family being studied. That evolution was followed up until the present epoch and circles fitted to their current distributions to find the forced and proper elements. Fig. 1b shows the outcome of several hundred runs showing the forced inclination of different sized dust from the Themis family (Kehoe et al. 2002). One outcome from these results was to show that while the particles are still in the $\mathrm{AB}$ their forced elements are essentially locked onto Jupiter, but passage through secular resonance at $\sim 2$ AU leads to a large dispersal in forced elements for different sized dust grains. This means that material contributing to the dust band peaks must be confined to $>2 \mathrm{AU}$.

The derived orbital element distributions were then converted into a 3D model of the spatial distribution of dust band material by choosing several hundred million orbits from these distributions and spreading the surface area around each orbit according to Kepler's laws. Observations through the dust bands were simulated by integrating the expected flux along a given line-of-sight making assumptions about the particles' emission properties and size distribution. The dust band components were compared with those observed using the same Fourier filtering methods (Fig. 1a) showing that by accounting for the full dynamical evolution of the dust grains the seasonal and wavelength variation of the bands can be accounted for (Grogan et al. 2001). Grogan et al. (2001) also found that the dust band shoulders are just the tip of the iceberg, and material in these bands contributes some $30 \%$ of the total ZC flux.

However, to fit the latitudes of the $\sim 10^{\circ}$ dust bands the proper inclination of the Eos family had to be artificially reduced to $9.35^{\circ}$. This has led to a revival of an interpretation 


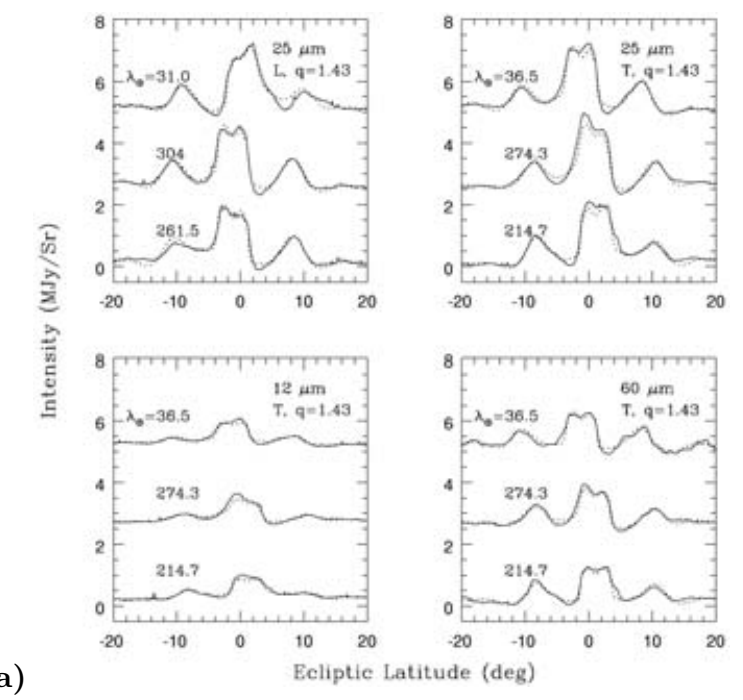

(b)
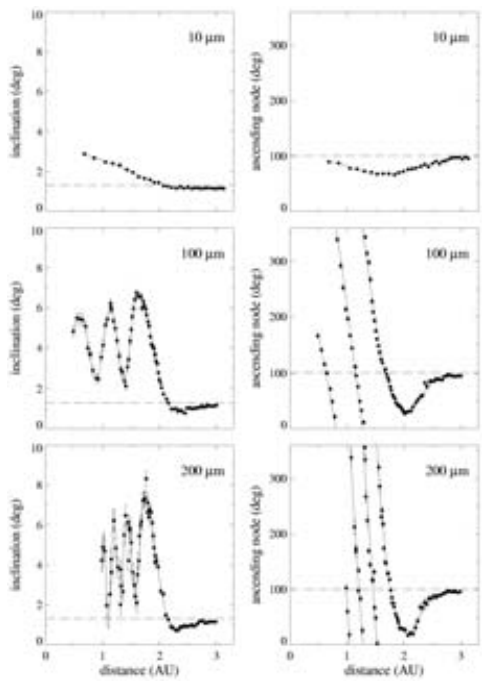

Figure 1. Dust band models. (a) Filtered IRAS dust band profiles (solid lines) at 12, 25 and $60 \mu \mathrm{m}$ compared with model profiles (Grogan et al. 2001). All profiles were made at $90^{\circ}$ solar elongation in the Leading (L) or Trailing (T) direction, and each figure shows the profiles at three different longitudes of the Earth. The models are made up of dust from the Eos, Themis and Koronis families with a size distribution defined by $q=1.43$. The distribution of the orbital elements of dust from each of the families was determined using the particle in a circle method to derive the forced and proper inclinations and eccentricities on different sized dust particles at different distances from the Sun at the epoch of the IRAS observations. (b) shows (left) the forced inclination as a function of semimajor axis and (right) the ascending node as a function of semimajor axis for dust of different sizes from the Themis family (Kehoe et al. 2002); Jupiter's osculating inclination and ascending node are shown with the dashed line.

originally proposed by Sykes \& Greenberg (1986), which is that the dust bands come from the recent break-up of individual asteroids (Dermott et al. 2002). Using new catalogues of asteroid proper elements Nesvorný et al. (2003) have been able to identify families (or indeed sub-families) which are at a suitable proper inclinations to form the bands: the $10^{\circ}$ band is now believed to originate in the break-up resulting in the Veritas family and the $2^{\circ}$ band is thought to originate in the Karin sub-family of the Koronis family (Nesvorný et al. 2002). Remarkably these are families believed to have formed just a few Myr ago. This new result will have a profound effect on our understanding of the ZC, since it implies that it is not in static equilibrium, rather that both its structure and brightness may vary significantly with time.

\subsection{Earth's Resonant Ring}

The other significant small scale structure in the ZC is the trailing/leading asymmetry. Both IRAS and COBE observations showed that the $\mathrm{ZC}$ is always brighter in the direction behind the Earth's motion than in front of it (Dermott et al. 1988; Reach et al. 1995). This asymmetry has been identified with dust trapped in resonance with the Earth (Dermott et al. 1994).

Resonance trapping is a well studied dynamical phenomenon (e.g., Jackson \& Zook 1989; Marzari \& Vanzani 1994; Beaugé \& Ferraz-Mello 1994). As dust grains migrate inward due to P-R drag they encounter the Earth's exterior mean motion resonances. Resonant forces can halt that migration by providing angular momentum to the dust grains, something which also increases the eccentricities of their orbits. Trapping is a probabilistic phenomenon and usually occurs into the first order $\mathrm{p}+1$ :p resonances which 
(a)

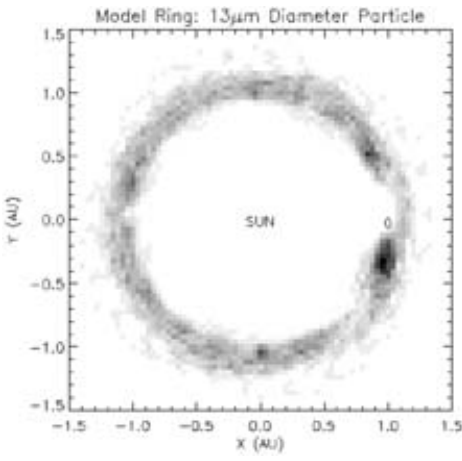

(b)

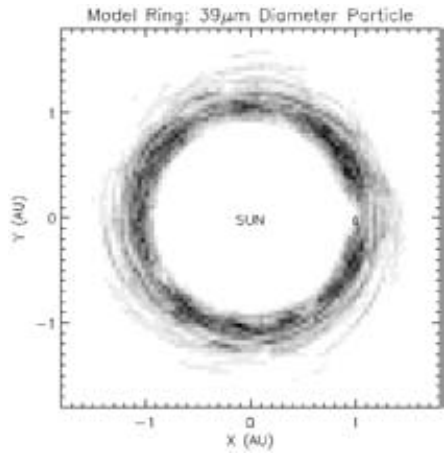

Figure 2. Face-on view of models for the Earth's resonant ring shown in the frame rotating with the Earth's mean motion (Dermott et al. 1994; Jayaraman \& Dermott 2004). The ring models are made up of asteroidal material of diameter (a) $13 \mu \mathrm{m}$ and (b) $39 \mu \mathrm{m}$ which are trapped into exterior mean motion resonance with the Earth. For both sizes the Earth, whose motion is shown with an ellipse at $x=1$ and $y=0$, resides in a cavity in the ring. Ring model structure includes a dense clump of material just below the Earth in these plots which is most prominent for smaller particle sizes.

are the strongest. It also occurs more readily for larger particles which move slower past the resonances. Trapping lasts typically for some tens of thousands of years, long enough to cause a significant density enhancement along the Earth's orbit, and particles eventually leave resonance after a close encounter with the Earth (Marzari \& Vanzani 1994). Trapping times are longer for larger particles as well as for resonances which are further from the planet (i.e., low $p$ resonances).

Dermott et al. (1994) constructed a 3D model for the structure of the Earth's resonant ring based on the results of simulations which determined the trapping probabilities and trapping durations for different sized dust grains migrating in from the $\mathrm{AB}$ past the Earth. A quantitative analysis of how the orbital elements are affected by the resonance was used to determine the distribution of orbital elements of material in the ring and the resulting model is shown in Fig. 2. This model has two defining features: a clump which follows the Earth around its orbit, and a cavity at the location of the Earth. These are features which are more clearly defined for small rather than large particles.

To explain why there is a clump and cavity in this model, consider the geometry of a particle on a 4:3 resonant orbit. If this particle is on an elliptical orbit then even without considering its dynamical interaction with the Earth, the particle will follow a loopy pattern in the frame rotating with the Earth and spend most of its time at 3 longitudes relative to the Earth (e.g., Murray \& Dermott 1999). Resonant forces from the Earth make the resonant argument of the particle, $\phi=(p+q) \lambda_{\mathrm{r}}-p \lambda_{\mathrm{pl}}-q \tilde{\omega}_{\mathrm{r}}$ (where $p+q=4$ and $p=3$ for the 4:3 resonance), librate about some value; this angle $\phi$ determines the orientation of the loopy pattern. For large bodies $\phi$ librates about $180^{\circ}$, which results in a pattern in which the loops (which correspond to locations where the particle is at pericenter) are as far from the Earth as possible and is symmetrical about the EarthSun line. For small dust, however, $\phi$ librates about an angle slightly higher than $180^{\circ}$ so that angular momentum can be transferred to the particle to stop it migrating out of resonance by $\mathrm{P}-\mathrm{R}$ drag. This means that the loopy pattern is asymmetric in the sense that the loop which is behind the Earth is closer to the Earth than the loop in front of it. The same is true for all resonances and explains why there is more material behind than in front of the Earth as well as why this asymmetry is stronger for smaller particles for which the drag force (and so asymmetry) is greater. 


\section{Dust in Extrasolar Systems}

The Sun is not the only star to have dust around it. In fact $15 \%$ of nearby stars are known to be surrounded by dust disks (e.g., Backman \& Paresce 1993). These were discovered by IRAS which showed that these stars exhibit more infrared emission than expected from the star alone (Aumann et al. 1984). The excess component emits at far-IR to sub-mm wavelengths and has a characteristic temperature of $<100 \mathrm{~K}$. It comes from dust grains heated by the star and its temperature implies that the dust is more than 30 AU from the stars. The dust is shortlived and so must be continually replenished, a fact which implies there are also planetesimals orbiting the star (Wyatt \& Dent 2002). The disks are thought to be the extrasolar equivalents to our own KB (Wyatt et al. 2003).

One of the main goals of subsequent studies has been to obtain images of these disks both to confirm the disk interpretation and to search for structure within them. To date some 10 or so of these disks have been imaged at wavelengths ranging from optical (Clampin et al. 2003), near-IR (Schneider et al. 1999), mid-IR (Telesco et al. 2000), submm (Holland et al. 1998) and mm (Wilner et al. 2002). These images confirm that these are rings of dust $>30 \mathrm{AU}$ from the star with holes at the center of similar size to the solar system. The images also show that the disks contain a variety of structures.

The interpretation of such structures is of fundamental importance to our understanding of the outcome of planetary formation in extrasolar systems. Not least because the identification of structure in the ZC with the solar system's planets shows that if there are planets in these disks then they would impose structure on the disks implying that the structures could be used to infer the presence of planets which would otherwise be undetectable. The challenge with modeling these structures is that there are essentially no constraints on the perturbing planetary systems meaning that detailed dynamical simulations are out of the question. Current modeling efforts focus on the ways in which different types of planetary perturbation affect the orbits of populations of planetesimals and dust. A vital prediction of any such model is how the resulting dynamical structures would be manifested in observations of the disks when parameters such as the mass and orbit of the perturbing planet are varied. The ultimate goal of a model is to be able to use observed structures to set constraints on the properties of unseen planets and to make predictions which can be tested with future observations.

\subsection{Offsets and Warps: Secular Perturbations}

Some of the ways in which planetary perturbations affect disk structure have been studied confirming not only that these perturbations are potentially observable, but that we may already have evidence of such perturbations in the current observations. The secular perturbations of a planet are a well studied example. If there is a planet on an eccentric orbit within a disk then its secular perturbations will impose a forced eccentricity on the orbits of all planetesimals near it. The effect on the planetesimal disk is to cause its center of symmetry to be offset from the star, a fact which makes one side of the disk hotter and therefore brighter than the other side (Wyatt et al. 1999). An asymmetry in the brightness of the lobes in the HR4796 disk has been detected (Telesco et al. 2000) which could be caused by a forced eccentricity as small as 0.02 (Wyatt et al. 1999). The planet's secular perturbations would also impose a forced inclination onto planetesimal orbits. Since this forced inclination defines the plane of symmetry of the disk, if this varies with distance, such as in a system with two or more planets on different orbital planes, then the disk will appear warped. The $\beta$ Pic disk, which is seen edge-on, has been observed to be significantly warped (e.g., Heap et al. 2000), an observation which has been modeled as due to planets on inclined orbits (e.g., Augereau et al. 2001). 


\subsection{Clumps: Collisions and Resonant Perturbations}

The most intriguing property of debris disks is that most of them are clumpy (Greaves et al. 1998; Holland et al. 2003). For example, a sub-mm image of the Vega disk, which has two clumps of asymmetric brightness embedded in it is shown in Fig. 3a (Holland et al. 1998). Taking the structure of the $\mathrm{ZC}$ as the basis for interpreting these clumpy structures, there are two possible models.

(a) Based on the origin of the dust bands, the first explanation is that the clumps are the products of recent individual collisions. While this remains a possibility, it is statistically unlikely. The problem is that most of these clumps have been detected in the sub-mm which means that the very fact that we are able to detect these clumps means that they contain so much mass that each clump would have to be the product of a collision between two planetesimals at least $1400 \mathrm{~km}$ in diameter (Wyatt \& Dent 2002). The interaction between such large planetesimals (e.g., Kokubo \& Ida 1995) means that there can only be $\sim 100$ such planetesimals in each disk, and the rate at which clumps would disperse into a ring after a collision means that they would last at most a few tens of thousands of years. Given the age of these disks of a few $100 \mathrm{Myr}$, we would have to be at a very special point in time to be witnessing the destruction of one of these planetesimals (Wyatt \& Dent 2002). The same may not be true, however, for the clump recently detected in the mid-IR observations of the $\beta$ Pic disk (Telesco et al. 2004), since such observations sample much lower dust masses and this system is only $\sim 10$ Myr.

(b) The other possibility is based on the Earth's resonant ring, i.e., that the clumps are resonant patterns caused by dust which has migrated into the resonances of an unseen planet. This interpretation has been pursued by several authors who have shown that structures similar to those observed in the disks around $\epsilon$ Eridani and Vega can be produced in this way if the perturbing planet is about the size of Jupiter or Saturn (Ozernoy et al. 2000; Wilner et al. 2002; Quillen \& Thorndike 2002; Kuchner \& Holman 2003). However, this interpretation has a serious problem which is that it ignores the effect of collisions. The collisional lifetime of particles in these disks is much shorter than their P-R drag lifetime. Using the terminology introduced in section 1.1, these are disks with $\eta_{0} \gg 1$. This means that mass flows through the collisional cascade so fast that asteroids are ground into dust which is removed by radiation pressure before P-R drag has had a chance to act (Wyatt et al. 1999). This problem was acknowledged by Wilner et al. (2002) who proposed that the parent planetesimals of the dust are in resonance.

(c) This led Wyatt (2003) to propose a third possibility which is based on the structure of the KB. Many objects in the KB are trapped in resonance with Neptune (e.g., Jewitt 1999). This resonant configuration is thought to be the result of resonance sweeping of the primordial KB which occurred when Neptune migrated out to its current location from an orbit closer to the Sun due to angular momentum exchange with the residual planetesimal disk left over after planet formation (Hahn \& Malhotra 1999). Such resonance sweeping may be a common feature of extrasolar planetary systems and the resulting resonant configuration in the KBs of extrasolar systems may be different to our own because of a different planet mass or migration rate.

Wyatt (2003) put together a model for the structure of planetesimal disks expected as a result of planet migration using techniques similar to those employed by Dermott et al. (1994). The model is based on the outcome of several hundred numerical simulations, each of which involving just 200 planetesimals, which were used to derive the probability of planetesimals becoming trapped into the 2:1, 5:3, 3:2 and 4:3 resonances as a result of the migration of different mass planets at different rates. The simulations were also used to derive the orbital element distributions of the trapped planetesimals. These results 


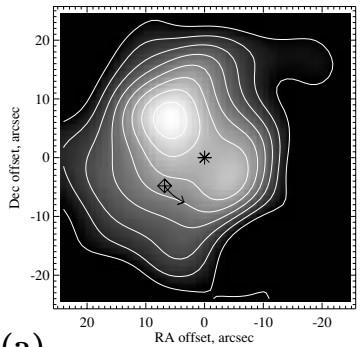

(a)

(a)
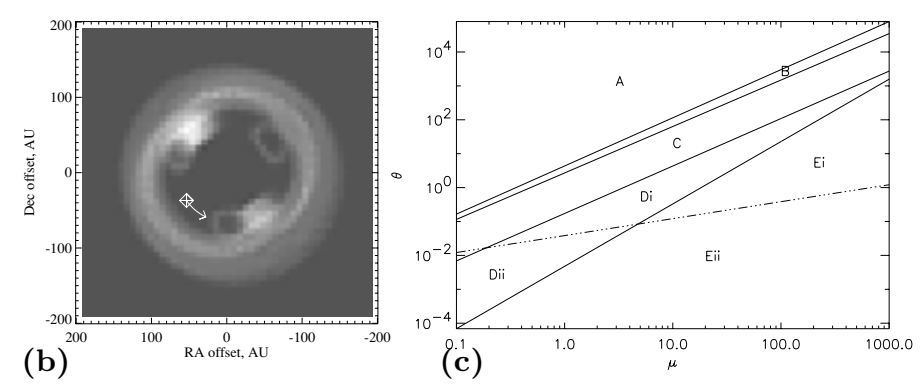

(c) the Vega disk (Holland et al. 1998). The star contributes little to the emission at this wavelength and is at the center of the image, halfway between two clumps of unequal brightness. (b) Structure of the underlying planetesimal disk in the model of Wyatt (2003) in which the clumps are explained by the outward migration of a planet which traps planetesimals into its resonances. (c) Phase space determining which resonances can be filled for migrations defined by $\mu$ (planet mass) and $\theta$ (migration rate) (Wyatt 2003). The solid lines show $50 \%$ trapping probability for the 4:3, 3:2, 5:3 and 2:1 resonances (top to bottom); the dash-dot shows the line for which twice as many planetesimals are trapped into the $2: 1(\mathrm{u})$ over the $2: 1(\mathrm{l})$ resonance.

were combined to make a 3D model of the spatial distribution of material resulting from any given planet migration. Assumptions were then made about the dust emission properties and an observing geometry simulated to get an image of the disk which was then convolved with the appropriate beam size to allow the model observation to be compared directly with that observed.

When this process was applied to Vega's clumpy disk it was found that the observed structure can be explained very well by a model in which a Neptune mass planet migrated from 40-65 AU over the course of 56 Myr. Such a planet traps planetesimals primarily into its 3:2 and 2:1(u) resonances which means that the spatial distribution of those planetesimals has two clumps on either side of the star of unequal brightness; the unconvolved image of the model is shown in Fig. 3b.

The most important feature of this modeling method was that it allowed constraints to be set on the planet causing the structure. This is because the model explored the structures caused by all possible planet masses and migration rates. The four different resonances which were studied have their own clumpy signature and trapping probabilities for the different resonances were found to be determined by the two parameters $\mu=M_{\mathrm{pl}} / M_{\star}$ and $\theta=\dot{a}_{\mathrm{pl}} \sqrt{a / M_{\star}}$ in the manner described in Fig. 3c (Wyatt 2003). Thus it was possible to say that the migration causing the Vega disk structure, which must come from the superposition of the $2: 1(\mathrm{u})$ and 3:2 resonances, must come from zone Di in Fig. 3c, although tighter constraints could be set on these parameters since the edges of the zones are soft and not hard as shown on this figure. So while the model proposed a Neptune mass planet taking 56 Myr to complete its migration, which is a migration strikingly similar to that proposed for our own Neptune, it is also possible that the planet was bigger and migrated faster and vice versa. The model also made testable predictions. such as that the structure will orbit the star with the planet at about $1^{\circ} /$ year, a level which we should be able to detect in a few years.

There is also a prediction of the model which may recently have been confirmed. The Wyatt (2003) model assumed that the dust seen in the images has the same orbital distribution as the large planetesimals in the disk. This is not necessarily true because of radiation pressure. As mentioned in section 1.1, radiation pressure means that dust grains see a lower mass star than their parent planetesimals which means the dust has a 
slightly different orbital period. To assess the impact of this effect more simulations were performed (Wyatt, in prep.) to follow the orbital evolution of dust grains with different levels of radiation pressure resulting from the destruction of resonant planetesimals. This showed that the dust grains do stay in resonance, but at the expense of an increased width of libration of $\phi$ for smaller grains. Since $\phi$ defines the orientation of the clumpy pattern, this causes the clumps to be smeared out azimuthally. The very smallest grains are no longer in resonance and have an axisymmetric distribution.

To find out whether this has any impact on the Wyatt (2003) model the spectral energy distribution of the Vega disk emission was studied to determine that the sizes of the dust grains contributing to the $850 \mu \mathrm{m}$ observations is between $300 \mu \mathrm{m}$ and $20 \mathrm{~cm}$ in diameter (e.g., Wyatt \& Dent 2002). Since the size at which dust falls out of resonance is $300 \mu \mathrm{m}-2$ mm depending on planet mass, the Wyatt (2003) model was correct in assuming that the dust distribution is similar to that of the planetesimals at $850 \mu \mathrm{m}$. However, this analysis also led to the prediction that mid- and far-IR observations of Vega will sample dust smaller than a few $\mathrm{mm}$, and since that dust is no longer in resonance its distribution should be smooth. In the last couple of months the Spitzer satellite has made images of the Vega disk at 25 and $70 \mu \mathrm{m}$ which show that the disk is indeed circularly symmetric at these wavelengths (Rieke et al. in prep.).

\section{Conclusions}

At least $30 \%$ of dust in the ZC comes from the break-up of asteroids. That dust migrates in toward the Sun by P-R drag, some of it getting broken up in collisions into smaller dust on the way. It also interacts with the planets causing structure and there is now evidence that the ZC may be sporadically replenished by the break-up of a few asteroids. Extrasolar systems also have dust belts, but these are more akin to dust coming from the $\mathrm{KB}$ than the $\mathrm{AB}$ in the solar system. The ones which have been detected so far are much more massive than the ZC, so their dynamics is different: both P-R drag and individual planetesimal collisions are much less important in shaping the disk. However, the disks do contain structures which may be evidence of unseen planets, both from the secular perturbations of those planets and clumpy resonant structure caused by the migration of planets.

\section{Acknowledgements}

I wish to acknowledge the Florida Solar System Dynamics Group (Stan Dermott et al.) whose work has contributed greatly to this paper.

\section{References}

Augereau, J.C., Nelson, R., Lagrange, A.M., Papaloizou, J. \& Mouillet, D. 2001, Astron. Astrophys., 370,447

Aumann, H.H., Gillett, F.C., Beichman, C.A., De Jong, T., Houck, J.R., Low, F.J., Neugebauer, G., Walker, R.G. \& Wesselius, P.R. 1984, Astrophys. J. 278, L23

Backman, D.E. \& Paresce, F. 1993, in E.H. Levy \& J. Lunine (eds.), Protostars and Planets III, (Tucson: Univ. Ariz. Press), p. 1253

Beaugé, C. \& Ferraz-Mello, S. 1994, Icarus, 110, 239

Burns, J.A., Lamy, P.L. \& Soter, S. 1979, Icarus, 40, 1

Clampin, M., et al. 2003, Astron. J., 126, 385

Dermott, S.F., Nicholson, P D., Burns, J.A. \& Houck, J.R. 1984, Nature, 312, 505

Dermott, S.F., Nicholson, P.D., Kim, Y., Wolven, B. \& Tedesco, E.F. 1988, in A. Lawrance (ed.), Comets to Cosmology, (Berlin: Springer-Verlag), p. 3 
Dermott, S.F., Gomes, R.S., Durda, D.D., Gustafson, B.Å.S., Jayaraman, S., Xu, Y.L. \& Nicholson, P.D. 1992, in S. Ferraz-Mello (ed.), Chaos, Resonance and Collective Dynamical Phenomena in the Solar System, (Dordrecht: Kluwer Acad. Publ.), p. 333

Dermott, S.F., Jayaraman, S., Xu, Y.L., Gustafson, B.A.S. \& Liou, J.-C. 1994, Nature, 369, 719

Dermott, S.F., Grogan, K., Durda, D.D., Jayaraman, S., Kehoe, T.J.J., Kortenkamp, S.J. \& Wyatt, M.C. 2001, in E. Grun, B.Å.S. Gustafson, S.F.Dermott \& H. Fechtig (eds.), Interplanetary Dust, (Heidelberg: Springer-Verlag), p. 569

Dermott, S.F., Kehoe, T.J.J., Durda, D.D., Grogan, K. \& Nesvorný, D. 2002, in B. Warmbein (ed.), Proceedings of Asteroids, Comets, Meteors (ACM 2002), (Noordwijk: ESA Publications Division), p. 319

Dohnanyi, J.S. 1969, J. Geophys. Res. 74, 2531

Durda, D.D. \& Dermott, S.F. 1997, Icarus, 130, 140

Durda, D.D., Greenberg, R. \& Jedicke, R. 1998, Icarus, 135, 431

Greaves, J.S., et al. 1998, Astrophys. J., 506, L133

Grogan, K., Dermott, S.F. \& Gustafson, B.Å.S. 1996, Astrophys. J., 472, 812

Grogan, K., Dermott, S.F., Jayaraman, S. \& Xu, Y.L. 1997, Planet. Space Sci., 45, 1657

Grogan, K., Dermott, S.F. \& Durda, D.D. 2001, Icarus, 152, 251

Gustafson, B.Å.S. 1994, Annual Rev. Earth Planet. Sci., 22, 553

Hahn, J.M. \& Malhotra, R. 1999, Astron. J., 117, 3041

Heap, S.R., et al. 2000, Astrophys. J., 539, 435

Holland, W.S., et al. 1998, Nature, 392, 788

Holland, W.S., et al. 2003, Astrophys. J., 582, 1141

Jackson, A.A. \& Zook, H.A. 1989, Icarus, 97, 70

Jayaraman, S. \& Dermott, S.F. 2004, Icarus, submitted

Jewitt, D.C. 1999, Annual Rev. Earth Planet. Sci., 27, 287

Kehoe, T.J.J., Dermott, S.F. \& Grogan, K. 2002, in S.F. Green et al. (eds.), Dust in the Solar System and Other Planetary Systems, (Amsterdam: Elsevier), p. 140

Kelsall, T., et al. 1998, Astrophys. J., 508, 44

Kokubo, E. \& Ida, S. 1995, Icarus, 114, 247

Kortenkamp, S.J. \& Dermott, S.F. 1998, Icarus, 135, 469

Kuchner, M.J. \& Holman, M.J. 2003, Astrophys. J., 588, 1110

Leinert, C. \& Grün, E. 1990, in R. Schween \& E. Marsch (eds.), Physics and Chemistry in Space - Space and Solar Physics, Vol. 20, (Berlin: Springer), p. 207

Love, S.G. \& Brownlee, D.E. 1993, Science, 262, 550

Low, F.J., et al. 1984, Astrophys. J., 278, L19

Marzari, F. \& Vanzani, V. 1994, Planet. Space Sci., 42, 101

Moro-Martín, A. \& Malhotra, R. 2002, Astron. J., 124, 2305

Murray, C.D. \& Dermott, S.F. 1999, Solar System Dynamics (Cambridge: Cambridge University Press)

Nesvorný, D., Bottke, W.F., Dones, L. \& Levison, H.F. 2002, Nature, 417, 720

Nesvorný, D., Bottke, W.F., Levison, H.F. \& Dones, L. 2003, Astrophys. J., 591, 486

Ozernoy, L.M., Gorkavyi, N.N., Mather, J.C. \& Taidakova, T.A. 2000, Astrophys. J., 537, L147

Quillen, A.C. \& Thorndike, S. 2002, Astrophys. J., 578, L149

Reach, W.T., et al. 1995, Nature, 374, 521

Schneider, G., et al. 1999, Astrophys. J., 513, L127

Sykes, M.V. \& Greeberg, R. 1986, Icarus, 65, 51

Sykes, M.V., Lebofsky, L.A., Hunten, D.M. \& Low, F.J. 1986, Science, 232, 1115

Telesco, C.M., et al. 2000, Astrophys. J., 530, 329

Telesco, C.M., et al. 2004, Nature, submitted

Wilner, D.J., Holman, M.J., Kuchner, M.J. \& Ho, P.T.P. 2002, Astrophys. J., 569, L115

Wyatt, M.C. 1999, PhD thesis, University of Florida

Wyatt, M.C., et al. 1999, Astrophys. J., 527, 918

Wyatt, M.C. \& Dent, W.R.F. 2002, Mon. Not. R. Astron. Soc. 334, 589

Wyatt, M.C., Holland, W. S., Greaves, J. S. \& Dent, W.R.F. 2003, Earth, Moon, Planets, 92, 423

Wyatt, M.C. 2003, Astrophys. J., 598, 1321 